\title{
In-situ Welding of Single-Walled Carbon nanotubes and Melting of Encapsulated Metal Clusters in Carbon Shells: Theory and Experiment
}

\author{
$\underline{\text { M. Terrones }}{ }^{\S}$, F. Banhart ${ }^{\#}$, E. Hernández ${ }^{\star}$, N. Grobert ${ }^{\sharp}$, J.C. Charlier ${ }^{*}$, H. Terrones ${ }^{\S}$ \\ and P.M. Ajayan $\%$ \\ $\S$ Advanced Materials Department, IPICyT, Venustiano Carranza 2425-A, 78210 San Luis Potosí, \\ SLP, México. \\ \# Universität Ulm, Zentrale Einrichtung Elektronenmikroskopie, 89069 Ulm, Germany. \\ \&Institut de Ciència de Materials de Barcelona (ICMAB-CSIC), Campus de Bellaterra, 08193 \\ Barcelona, Spain \\ ${ }^{¥}$ Fullerene Science Centre, University of Sussex, Brighton BN1 9QJ, UK. \\ ${ }^{*}$ Université Catholique de Louvain, UPCPM, B-1348 Louvain-la-Neuve, Belgium. \\ ${ }^{\%}$ Department of Materials Science \& Engineering, Rensselaer Polytechnic Institute, Troy, NY \\ 12180-3590, USA.
}

The remarkable properties of carbon nanotubes and nanoparticles such as fullerenes, nanotubes, or carbon onions are mainly based on the extreme strength and rigidity of the covalent $\mathrm{C}-\mathrm{C}$ bond within the graphite lattice [1].

Regarding single-walled carbon nanotubes (SWNTs), little progress has been reported on techniques related to connecting such tubular structures. The latter is a key issue because both electronic devices and strong nano-mechanical systems need the establishment of molecular connections among SWNTs. Here, we demonstrate for the first time, that irradiation exposure at elevated temperatures, can be used as an effective tool to covalently weld SWNTs in order to create molecular junctions of various geometries. We have fabricated "Y", "X" and "T-like" junctions (Fig. 1), that are stable [2]. Tight binding molecular dynamics calculations demonstrate that vacancies, formed under the electron beam, trigger the formation of molecular junctions involving seven or eight membered carbon rings (Fig. 2). We envisage that our results will pave the way towards controlled fabrication of novel nanotube based molecular circuits, nanotube fabrics and network architectures.

In nanocomposites of graphitic structures and metal crystals, the extreme properties of graphite can be made use of to modify the shape and physical properties of small metal crystals. We also show, for the first time, that the melting and solidification behavior of metal crystals can be drastically altered when they are encapsulated in fullerene-like graphitic shells [3]. The melting temperature of low metling point metal crystals (e.g. $\mathrm{Bi}, \mathrm{Sn}, \mathrm{Pb}$, etc.) inside graphitic shells is increased relative to the bulk melting point by a much larger amount than that observed for metal crystals embedded in other materials. It appears that graphite is the ultimate material for enhancing the melting/solidification hysteresis of small crystals or clusters. Therefore, metal clusters encapsulated by graphitic shells may be potentially advantageous in temperature-resistant crystalline composite materials.

\section{References}

[1] M. Terrones, et al., Top. In Curr. Chem. 199 (1999) 189.

[2] M. Terrones, et al., Phys. Rev. Lett. (2002) 89, 75505.

[3] F. Banhart, E. Hernández, M. Terrones. Submitted (2003). 

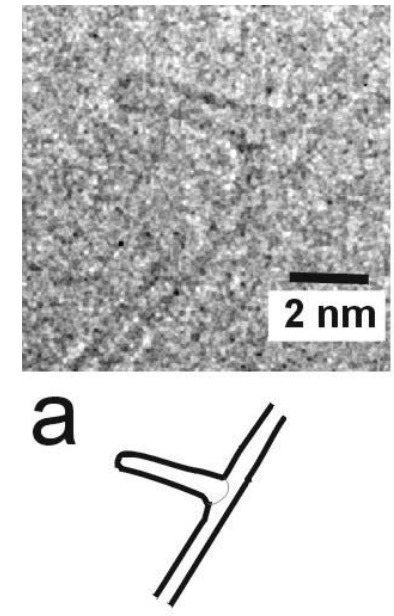

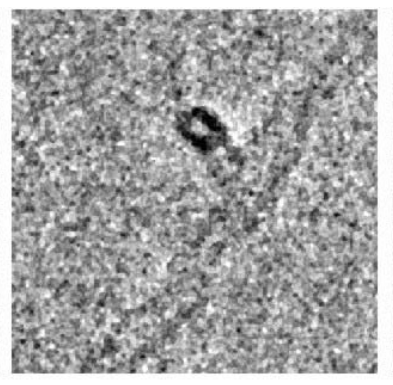

b

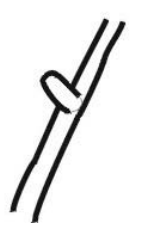

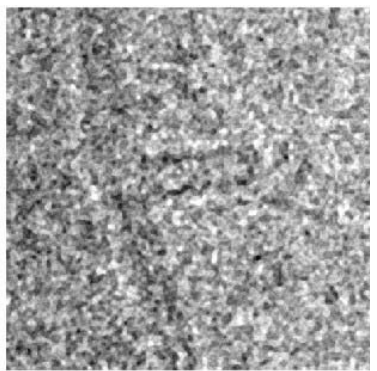

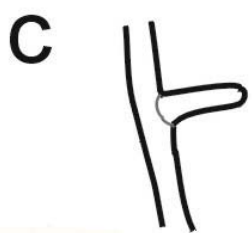

FIG. 1 (a-c) HRTEM images of a "T-like" junction formed after irradiating a "Y" junction. It is possible to thin one of the tubes of the "Y" junction, which eventually breaks and forms a "hook" (not shown here). It is also possible to observe the evolution of this junction during irradiation and the rotation by $180^{\circ}$ under the electron beam; note the circular cross-section in one of the tubes (b).

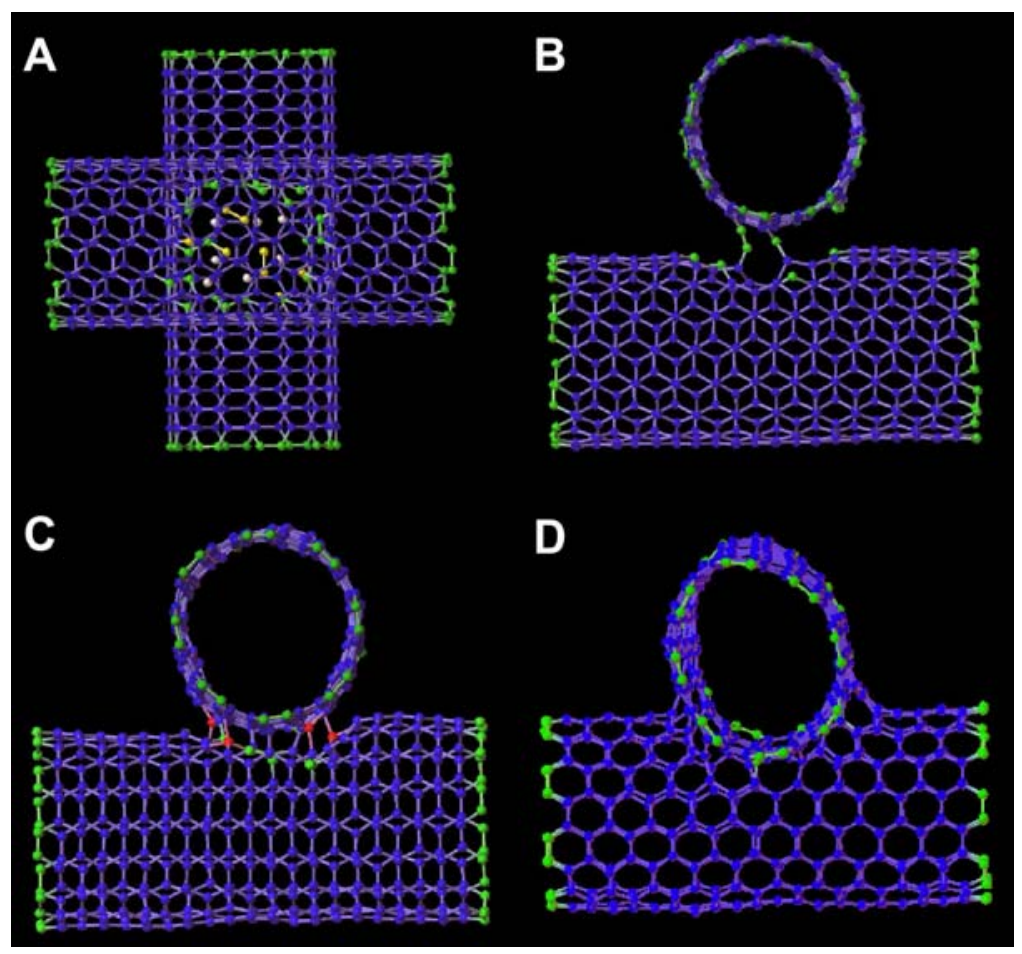

FIG. 2 TBMD simulations at $1000^{\circ} \mathrm{C}$ of two crossing $(8,8)$ nanotubes, which transform into an " $\mathrm{X}$ " junction: (a) Two crossing tubes containing 20 vacancies on their surfaces (carbon atoms in white, yellow, green, blue and red, illustrate atomic coordination of 0, 1, 2, 3, and 4, respectively); (b) After 10ps, two links between the two defected carbon structures are formed via carbon chains; (c) After $100 \mathrm{ps}$, a surface reconstruction occurs and the resulting structure consists of a molecular " $\mathrm{X}$ " junction; (d) After 220ps, a surface reconstruction occurs and the resulting structure consists of a molecular "X" junction. This reconstructed surface mainly contains $s p^{2}$ carbon atoms, and exhibit six heptagons, one octagon, one pentagon and two dangling bonds. 